(1985) Pferdeheilkunde 1, 65-69

\section{Olfaktorius-Neuroblastom bei einem Pferd}

\author{
G. Loupal, Marina Mikula
}

Aus dem Institut für Pathologie und Gerichtliche Veterinärmedizin (Vorstand: o. Univ.-Prof. Dr. H. Köhler) der Veterinärmedizinischen Universität Wien und der tierärztlichen Praxis Tzt. Marina Mikula, Wien-Liesing

Über spontane neuroepitheliale Tumoren der Riechschleimhaut bei Haussäugetieren ist bisher kaum berichtet worden. Bei zwei Katzen fanden Pospischil und Dabme (1981) Olfaktorius-Neuroblastome (Ästhesioneuroblastome), Anderson und Cordy (1981) beobachteten eine ähnliche Geschwulst bei einer 14 Monate alten Hereford-Kalbin. Experimentell konnten neuroepitheliale Tumoren der Riechschleimhaut mit Diäthylnitrosamin bei Goldhamstern (Herrold, 1964) sowie mit verschiedenen Nitrosaminen bei Ratten (Thomas, 1965) erzeugt werden.

Obwohl beim Menschen seit der Erstbeschreibung eines neuroepithelialen Tumors der Riechschleimhaut durch Berger und Mitarb. (1924) zahlreiche Mitteilungen verfaßt wurden, sind sie auch beim Menschen „derartig selten, daß niemand mit ihnen wirklich vertraut werden kann" (Gerard-Marchant und Micheau, 1965). Der Ursprung dieser Neoplasmen liegt im Riechepithel (Obert und Mitarb., 1960; Skolnik und Mitarb., 1966). Somit finden sich die allermeisten dieser Geschwülste im Bereich der Regio olfactoria, von wo sie sich in angrenzende Areale (z. B. Nasennebenhöhlen oder durch die Siebplatte ins Riechhirn) ausbreiten. Nach Gerard-Marchant und Micheau (1965) unterscheidet man zwischen Ästhesioneuroepitheliomen, Ästhesioneurozytomen und Ästhesioneuroblastomen. Letztere sind die am wenigsten differenzierte Form. Als maligne Geschwülste müssen aber alle genannten angesehen werden (Gerard-Marchant und Micheau, 1965). Infiltration und Zerstörung von Bindegewebe und Muskulatur sind bei ihnen häufig (Skolnik und Mitarb., 1966).

Das Olfaktorius-Neuroblastom ist histologisch charakterisiert durch Nester von neoplastischen Zellen, die durch viele Gefäße enthaltende Bindegewebssepten getrennnt sind. Die Zellen haben wenig Zytoplasma mit ausgesprochen undeutlichen Zellgrenzen und runde bis ovale Kerne. Sie ordnen sich mitunter zu echten Rosetten oder Pseudorosetten an. Zwischen den Zellen finden sich Neurofibrillen (Obert und Mitarb., 1960; Noltenius, 1981). Da die histologische Diagnose eines Olfaktorius-Neuroblastoms sehr schwierig sein kann, wird empfohlen, das Elektronenmikroskop zu Hilfe zu nehmen (Taxy und Hidvegi, 1977). Als ultrastrukturelles Charakteristikum werden vor allem neurosekretorische Granula angesehen (Osamura und Fine, 1975 und 1976; Taxy und Hidvegi, 1977; Wilander und Mitarb. 1977; Pospischil und Dabme, 1981). Daneben werden noch als Besonderheiten Neurotubuli und Neurofilamente gefunden

\section{Zusammenfassung}

Bei einem 16 Jahre alten holländischen Warmblut-Wallach mit Nasenbluten und rechtsseitigem Exophthalmus wurde bei der Sektion des Kopfes ein Tumor im Bereich des Nasengrundes der rechten Seite, der sich auch in die rechte große Kieferhöhle sowie in die rechte Gaumenkeilbeinhöhle erstreckte, gefunden. Beide Nebenhöhlen waren außerdem hochgradig mit geronnenem Blut gefüllt. Durch die histologische Untersuchung und insbesondere durch den elektronenmikroskopischen Nachweis von neurosekretorischen Granula wurde die Diagnose Olfaktorius-Neuroblastom gestellt. Der vorliegende Fall stellt eine Erstbeschreibung eines derartigen Tumors beim Pferd dar.

\section{An olfactory neuroblastoma in a horse}

When dissecting the head of a 16 year old Dutch Standardbred gelding, a tumor was found in the nasal fundus region of the right-hand side, also involving the large maxillary sinus as well as the palatinosphenoidal sinus on the right. Furthermore, both the paranasal sinuses were filled up with cloded blood. An olfactorius neuroblastoma was diagnosed using histological examination and especially evidence of neurosecretory granules in the electron microscope. This is the first time this kind of tumor is being described in a horse.

(Osamura und Fine, 1976; Taxy und Hidvegi, 1977; Pospischil und Dabme, 1981). Die vorliegende Kasuistik stellt eine Erstbeschreibung eines Olfaktorius-Neuroblastoms beim Pferd dar.

\section{Klinischer Befund}

Am 2. Juni 1984 wurde in der Praxis ein 16jähriger Fuchswallach, holländisches Warmblut, mit einseitigem Nasenbluten vorgestellt.

Die klinische Untersuchung ergab neben einem geringgradig schmerzhaften Lymphonodus mandibularis dexter eine geringgradige, einseitige kontinuierliche Blutung aus dem rechten Nasengang, die sich bei Senkung des Kopfes etwas verstärkte. Weitere pathologische Befunde konnten nicht erhoben werden. Ein Endoskop konnte nur ca. $10 \mathrm{~cm}$ in den rechten ventralen Nasengang eingeführt werden, da dieser hochgradig eingeengt war.

Im Röntgenbild konnte eine homogene Verschattung im rostralen und kaudalen Teil des Sinus maxillaris festgestellt werden. Die Verschattung war nach dorsal hin gerade und horizontal begrenzt. Wir stellten die Diagnose: Erguß in der Kieferhöhle.

$\mathrm{Da}$ die Blutungsätiologie unbekannt war (Folgen eines Traumas konnten mit Sicherheit ausgeschlossen werden) wurde symptomatisch therapiert. Nach einer intensiven zehntägigen Therapie mit Hämostyptika, Bestrahlung mit einer Infrarotlampe und Bodenfütterung kam die Blutung bald zum Stillstand. Das Kontrollröntgen nach drei Wochen zeigte eine fast vollständige Entleerung des Sinus maxillaris. Bodenfütterung und Bestrahlung wurden noch zwei Wochen weitergeführt und das Pferd anschließend der normalen Arbeit zugeführt.

Zwei Monate später fielen dem Besitzer Veränderungen im Verhalten (vor allem wurde das Pferd besonders schreckhaft), Ataxien und unsicherer Gang auf. 
Die erneute klinische Untersuchung zeigte wieder eine geringgradige, aber diesmal diskontinuierliche Blutung aus dem rechten Nasengang, zusätzlich am rechten Auge einen beginnenden Exophthalmus, verstärkte Gefäßinjektion der Skleralgefäße und fehlenden Pupillarreflex (Pupille weit und starr). Der Sehfunktionstest bestätigte den Verdacht der Erblindung dieses Auges. Nach einer Woche erblindete auch das linke Auge. Das Nichtansprechen auf jegliche Therapie und das offensichtlich rasche Fortschreiten der Erkrankung erhärtete den Verdacht auf einen Tumor, worauf der Besitzer einer Euthanasie zustimmte.

Nach der Tötung wurde der Kopf des Pferdes im AlantoOkzipital-Gelenk abgesetzt und dem Institut für Pathologie und Gerichtliche Veterinärmedizin zur Untersuchung überbracht.

\section{Makroskopischer Befund}

Zur makroskopischen Untersuchung wurde der Pferdeschädel annähernd in der Medianlinie auseinandergesägt und das an der rechten Schädelhälfte verbliebene Septum nasi entfernt. Danach wurden die einzelnen Nasennebenhöhlen der Reihe nach untersucht. Im Bereich des Nasengrundes der rechten Seite, in den kaudalen Abschnitten der rechten großen Kieferhöhle sowie in den rostralen Anteilen der rechten Gaumenkeilbeinhöhle fand sich ein rosafarbenes, speckig glänzendes Gewebe, das an den knöchernen Wänden derartig fest anhaftete, daß es nur unvollständig aus den Höhlen entfernt werden konnte. Offensichtlich hatte dieses Gewebe den Knochen infiltriert. Das Gewebe war weich und sehr brüchig. In den übrigen Anteilen der rechten großen Kieferhöhle sowie der rechten Gaumenkeilbeinhöhle war in großer Menge geronnenes Blut, das sich zum Unterschied von dem oben beschriebenen Gewebe sehr leicht aus den Höhlen entfernen ließ. Sowohl die große Kieferhöhle als auch die Gaumenkeilbeinhöhle waren somit vollständig mit Blutkoagula bzw. dem speckigen rosafarbenen Gewebe gefüllt. Eine scharfe Trennlinie zwischen diesen beiden Anteilen konnte nicht nachgewiesen werden. Das Siebbeinlabyrinth war im Bereich der Gewebsmassen teilweise destruiert, die Siebplatte war unverändert. Ein Durchwachsen des Gewebes durch das Siebbein in die Schädelhöhle konnte nicht festgestellt werden. Die übrigen Nasennebenhöhlen sowie der Rest der Nasenhöhle waren ebenso ohne Besonderheiten wie die Lymphknoten. Auch die Augen und das Gehirn waren makroskopisch unauffällig, lediglich in den beiden Seitenventrikeln konnten jeweils kleinbohnengroße Plexuscholesteatome (in jedem Seitenventrikel eines) gefunden werden.

\section{Histologischer Befund}

Mehrere Teile des makroskopisch nachgewiesenen Gewebes im Nasengrund sowie in den beiden Nebenhöhlen wurden in 10prozentigem Formalin fixiert und in Paraffin eingebettet. Nach Anfertigung von ca. $5 \mu \mathrm{m}$ dicken Schnitten wurden folgende Färbemethoden angewandt: HE, Silbermethoden nach Bodian, Grimelius und Sevier-Munger. Weiters wurden mehrere Lokalisationen des Gehirns und des rechten Auges histologisch untersucht (Fixierung und Einbettung wie oben, HE-Färbung).

Bei der Untersuchung des Gewebes vom Nasengrund fiel sofort auf, daß es sich um einen Tumor handelte. Das in den Nebenhöhlen gefundene Gewebe war histologisch völlig gleichartig. Das Neoplasma bestand aus locker gefügten Zellen, die in einem Netz aus sehr gefäßreichem Bindegewebe lagen. Meist waren die durch das fibrovaskuläre Stroma gebildeten Räume, in denen sich die Tumorzellen befanden, rund oder oval (Abb. 1). Die Tumorzellen selbst hatten runde, chromatindichte, mehr oder weniger an Lymphozyten erinnernde Kerne. Seltener waren auch ovale, etwas chromatinärmere Kerne zu sehen. Eine Kernpolymorphie war nur mäßig ausgeprägt, Kernteilungsfiguren waren praktisch nicht nachzuweisen. Die Zellen hatten wenig eosinophiles Zytoplasma, die Zellgrenzen waren undeutlich. Bei sehr vielen Zellen fielen mehrere zipfelartige Zytoplasmaausläufer auf, mit denen sie untereinander in Verbindung zu stehen schienen. In mehreren Lokalisationen sahen wir Fibrillen, die zwischen den Zellen lagen bzw. manchmal auch aus den Zytoplasmaausläufern hervorgingen (Abb. 2). Echte Rosetten konnten wir in dem

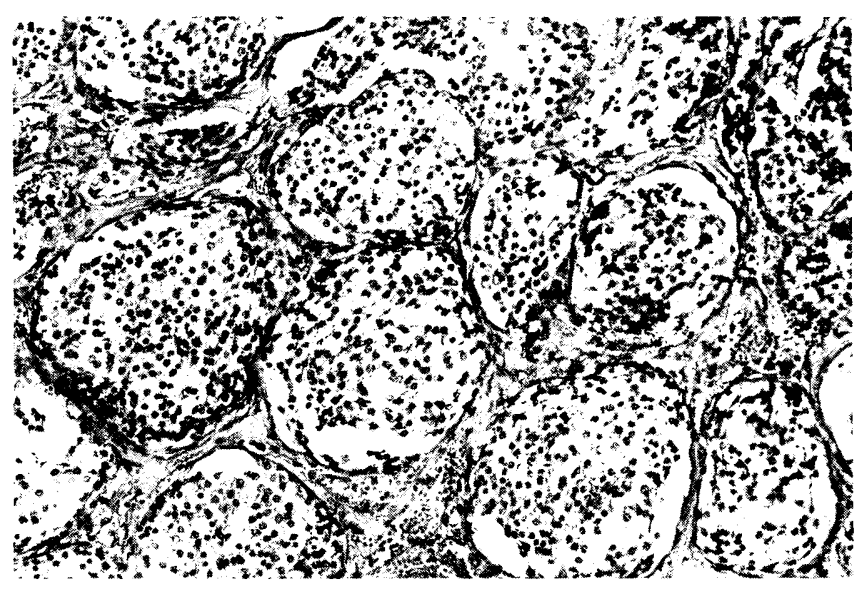

Abb. 1: Olfaktorius-Neuroblastom; Mikrophoto, HE-Färbung, schwa ches Trockensystem

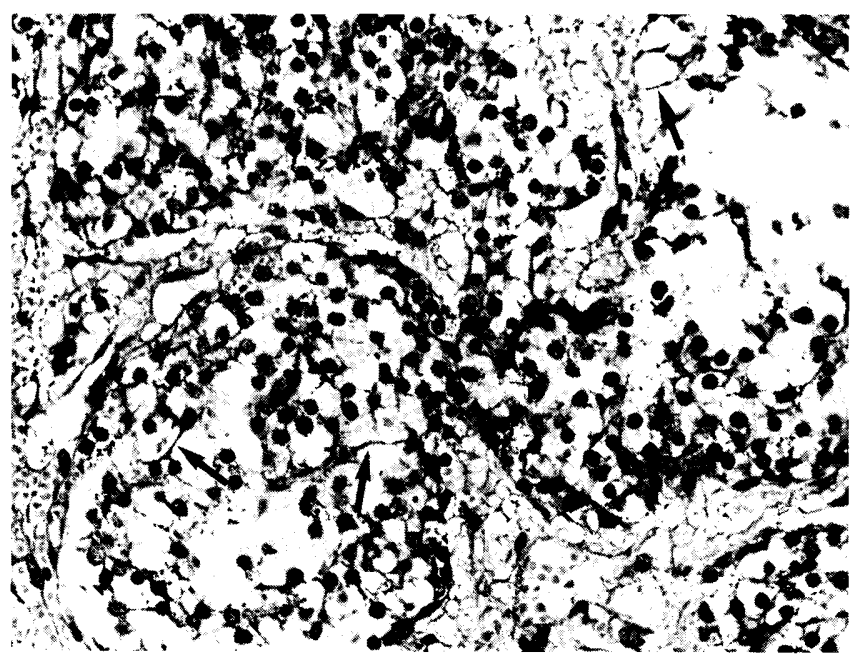

Abb. 2: Olfaktorius-Neuroblastom, Neurofibrillen (Pfeile); Mikrophoto, Silbermethode nach Sevier-Munger, starkes Trockensystem 


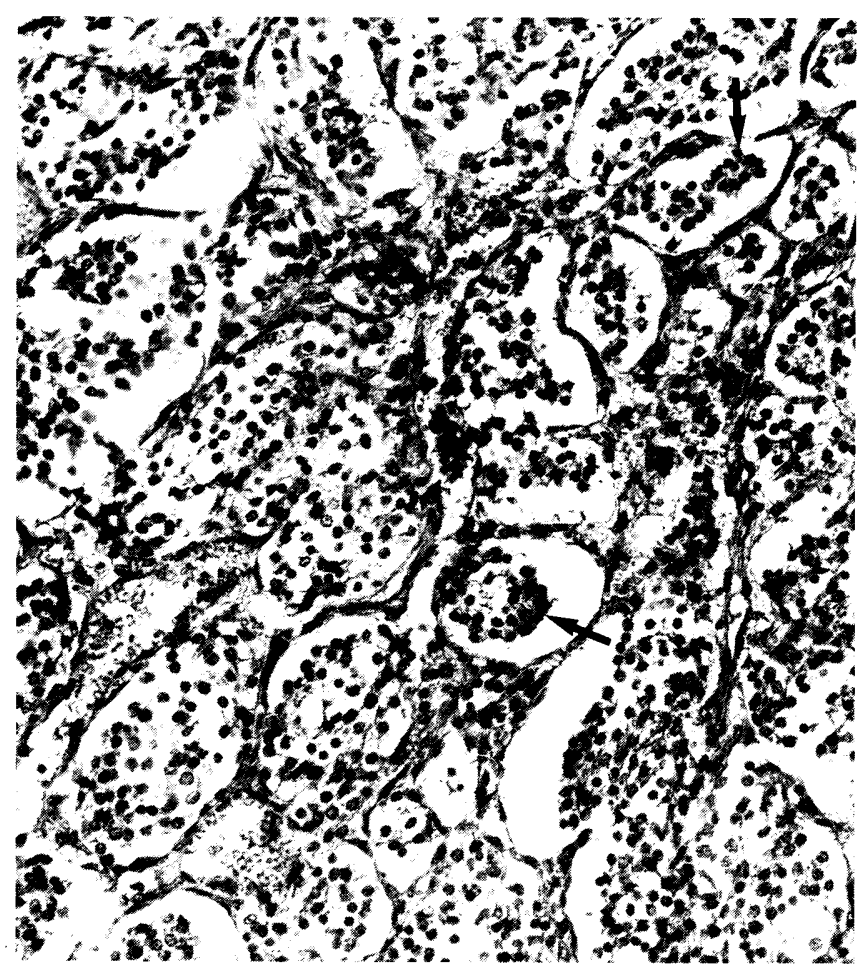

Abb. 3: Olfaktorius-Neuroblastom, Pseudorosetten (Pfeile); Mikro photo, HE-Färbung, mittleres Trockensystem

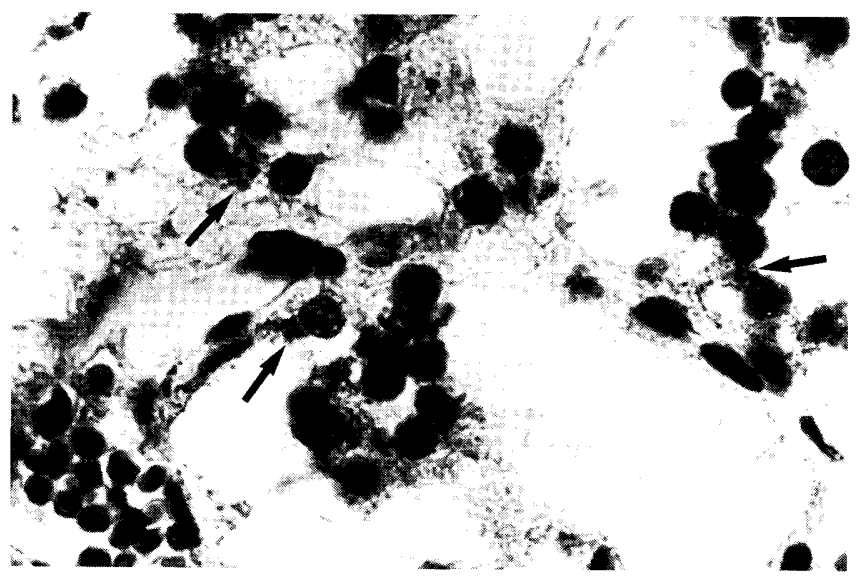

Abb. 4: Olfaktorius-Neuroblastom, argyrophile Granula (Pfeile); $M$ krophoto, Silbermethode nach Grimelius, Ölimmersion

Tumor nicht nachweisen, Pseudorosetten waren an mehreren Stellen zu erkennen (Abb. 3)

Der Tumor wuchs infiltrativ. In vielen Lokalisationen waren Blutungen zu sehen, die mancherorts große Ausdehnung erlangten. Nekrosen fehlten in den untersuchten Lokalisationen.

Mit allen angewandten Silbermethoden ließen sich die Fibrillen gut darstellen, wobei die besten Erfolge mit der Methode nach Sevier-Munger erzielt wurden. Bei der Methode nach Grimelius ließen sich sogar argyrophile Granula in einigen wenigen Zellen erkennen (Abb. 4).

Durch die histologische Untersuchung des rechten Auges, seines Nervus opticus und des Chiasma opticum konnten keine Besonderheiten ermittelt werden. Bei der Untersuchung des Gehirns konnte auch histologisch ein Eindringen des Tumors in das Riechhirn nicht nachgewiesen werden. Eine vermehrte Lipofuszinspeicherung in Ganglienzellen war vor allem in Mittelhirn und Medulla oblongata zu erkennen. Einzelne Purkinjezellen im Kleinhirn wiesen degenerative Veränderungen auf (Homogenisierung und Vakuolisierung des Zytoplasmas bei regressiven Kernveränderungen), manche waren dystrophisch verkalkt. Sonst waren im Gehirn auch histologisch keine Besonderheiten zu entdecken.

\section{Elektronenmikroskopischer Befund}

Kleine Teile des formalinfixierten Tumors wurden für mehrere Stunden in Millonig-Puffer gebracht, dann zwei

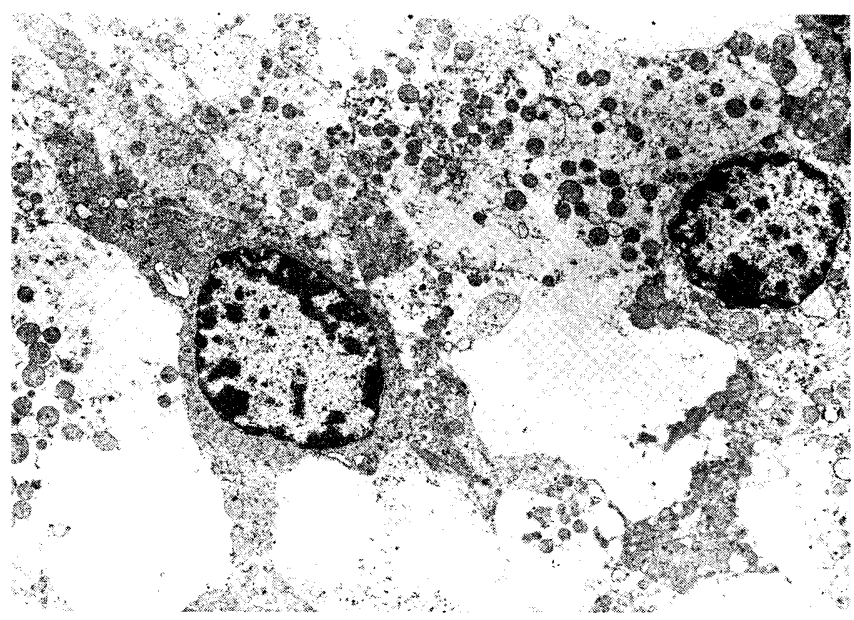

Abb. 5: Olfaktorius-Neuroblastom; elektronenmikroskopische Aufnahme, 5040x

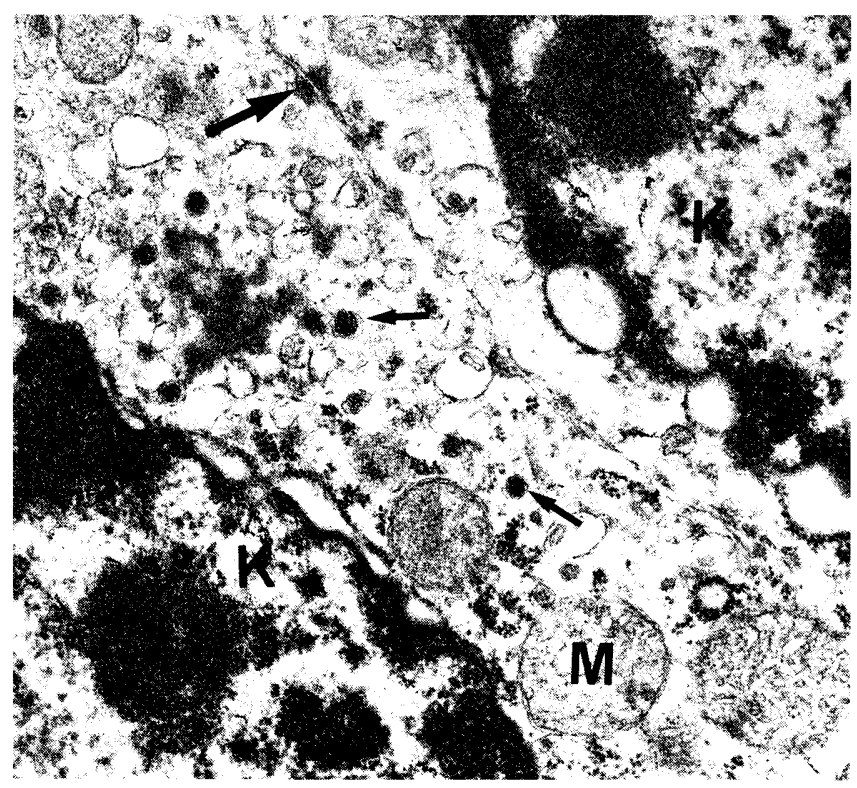

Abb. 6: Olfaktorius-Neuroblastom, neurosekretorische Granula (kleine Pfeile), Desmosom (großer Pfeil), Mitochondrien (M), Kerne (K); elektronenmikroskopische Aufnahme, 35000x 
Stunden in Osmiumtetroxyd nachfixiert und schließlich in einer aufsteigenden Alkoholreihe entwässert. Nach EponEinbettung, Anfertigung von Ultradünnschnitten und Kontrastierung mit Uranylazetat und Bleizitrat wurden die Präparate im Elektronenmikroskop (Philips EM 400) durchgemustert.

Da nur mehr formalinfixiertes Tumorgewebe verfügbar war, konnte keine optimale Präparation für die Elektronenmikroskopie erfolgen. Da das Pferd aber ca. 2 Stunden nach der Schlachtung zur pathologischen Untersuchung gebracht worden war, waren die Zellen trotzdem noch relativ gut erhalten.

Die Zellen hatten, wie bereits lichtmikroskopisch festgestellt, runde bis ovale Kerne und einige Zytoplasmaausläufer (Abb. 5). Zwischen den Zellen fanden sich einige Fortsätze anderer Zellen. An Zellorganellen ließen sich zahlreiche Mitochondrien, wenig rauhes endoplasmatisches Retikulum und einige freie Ribosomen nachweisen. Vielerorts fanden sich einzelne neurosekretorische Granula, die sich durch ein elektronendichtes Zentrum und einen hellen Hof auszeichneten (Abb. 6). Zwischen einigen Zellen waren desmosomale Haftkomplexe ausgebildet (Abb. 6). Für eine deutliche Darstellung von Neurotubuli und Neurofilamenten war das Material nicht mehr gut genug erhalten. Mancherorts konnten dennoch für Neurotubuli verdächtige Strukturen ausgenommen werden.

\section{Diskussion}

Da das lichtmikroskopische Bild des von uns vorgestellten Tumors weitgehend die von Obert und Mitarb. (1960) erhobenen Charakteristika für Olfaktorius-Neuroblastome erkennen läßt (nur echte Rosetten konnten wir nicht finden) und da vor allem elektronenmikroskopisch neurosekretorische Granula nachweisbar sind, läßt sich unsere Diagnose absichern.

Beim Menschen unterscheiden Gerard-Marchant und Micheau (1965), wie oben erwähnt, zwischen Ästhesioneuroepitheliomen, -neurozytomen und -neuroblastomen. Bei ersteren stehen Rosetten und kompakte Zellgruppen mit Nervenfasern im Vordergrund des histologischen Bildes. Ästhesioneurozytome sind im wesentlichen gekennzeichnet durch regelmäßige säulenartige Anordnung von $\mathrm{Tu}$ morzellen entlang von Nervenfasern. Die Diagnose von Ästhesioneuroblastomen basiert nach Gerard-Marchant und Micheau (1965) meist auf negativen Kriterien, nämlich dem Fehlen von Rosetten und dem Fehlen der üblichen histologischen Struktur der Ästhesioneurozytome. Auch nach diesen Gesichtspunkten läßt sich die von uns beschriebene Geschwulst als Olfaktorius-Neuroblastom klassifizieren.

Von den bisher bei Haussäugetieren beschriebenen Olfaktorius-Neuroblastomen (Pospischil und Dabme, 1981; An. derson und Cordy, 1981) unterscheidet sich das histologische Bild des Tumors beim Pferd in einigen Punkten. So fielen bei den anderen Fällen die von uns relativ häufig beobachteten zipfeligen Zytoplasmaausläufer der neoplastischen Zellen nicht auf. Auch scheinen in unserem Fall mehr Fibrillen zwischen den Tumorzellen vorzukommen.
Echte Rosetten fehlten wie bei unserem Pferd bei der Geschwulst beim Rind (Anderson und Cordy, 1981), wurden jedoch bei den Katzen von Pospischil und Dabme (1981) gefunden. Die von uns angewandten Silbermethoden erwiesen sich in der Diagnostik des Olfaktorius-Neuroblastoms als sehr hilfreich. Mit ihnen ließen sich die Neurofibrillen gut darstellen. Mit der Methode nach Grimelius gelang es uns sogar, argyrophile Granula nachzuweisen, was auch beim Menschen mitunter gelang (Wilander und Mitarb., 1977).

Dem elektronenmikroskopischen Nachweis von neurosekretorischen Granula kommt die größte Bedeutung bei der Diagnose eines Olfaktorius-Neuroblastoms zu (Wilander und Mitarb., 1977; Noltenius, 1981; Pospischil und Dab$m e, 1981)$. Aber auch andere ultrastrukturelle Details unseres Tumors stimmen mit Beschreibungen von humanen oder felinen Olfaktorius-Neuroblastomen überein. So wurden zytoplasmatische Fortsätze (Neuriten) auch von Taxy und Hidvegi (1977) und von Taxy und Battifora (1980) beschrieben und Desmosomen auch von Taxy und Hidvegi (1977) sowie von Pospischil und Dabme (1981) gefunden. Für einen sicheren Nachweis von Neurotubuli und Neurofilamenten war unser Material infolge Formalinfixierung nicht mehr gut genug erhalten. Wir stimmen aber mit Osamura und Fine (1976) darin überein, daß eine elektronenmikroskopische Untersuchung auch von formalinfixierten $\mathrm{Ge}$ websproben bei der Klassifizierung von lichtmikroskopisch nicht zuzuordnenden Tumoren als Neoplasmen neuroepithelialen Ursprungs sinnvoll ist.

Die Krankheitssymptome des Pferdes ließen sich durch den Nachweis des Olfaktorius-Neuroblastoms zum Teil erklären. Das Nasenbluten sowie der einseitige Exophthalmus sind auf das Neoplasma zurückzuführen. Mit Rücksicht auf den Reichtum der Geschwulst an Blutgefäßen ist eine massive Blutung aus dem Tumor durchaus zu erwarten. Die Erblindung sowie die zentralnervalen Symptome wie die Verhaltensänderungen, die Ataxien und der unsichere Gang konnten morphologisch nicht abgeklärt werden. Der Tumor war nicht, wie man dies hätte erwarten können, durch die Siebplatte in das Gehirn eingedrungen. Der Druck, der durch das Neoplasma im Bereich des Nasengrundes auf die umgebenden Gebilde ausgeübt wurde, mag aber durchaus eine Erklärung für die zentralnervalen Störungen darstellen. Auch die degenerativen Veränderungen von einigen Purkinjezellen des Kleinhirns, über deren Ursache wir keine sichere Aussage treffen können, dürften bei der Entstehung der zentralnervalen Störungen eine Rolle gespielt haben.

Massive Epistaxis in Verbindung mit einseitigem Exophthalmus muß auch beim Pferd an einen Tumor in der $\mathrm{Na}$ senhöhle denken lassen. An sich sind Geschwülste der Nasenhöhle bei unseren Haussäugetieren selten. Vor allem finden sich Neoplasien des Oberflächenepithels oder der Drüsen der Nasenhöhle (Papillome, Plattenepithelkarzinome, Adenome, Adenokarzinome) sowie seltener mesenchymale Geschwülste (Stünzi und Hauser, 1976).

Aus früheren Jahren liegen auch Berichte über endemische Geschwülste des Siebbeins bei Rindern und Pferden in Schweden vor (Magnusson, 1916). Die ansteckenden Tumo- 
ren sollen teils karzinomatösen, teils sarkomatösen Aufbau gehabt haben. Beim Schaf gibt es gleichfalls Geschwülste, die von der Riechschleimhaut ihren Ausgang nehmen und übertragbar sind (Adenopapilloma infectiosum - Cobrs,

\section{Literatur}

Anderson, B. C., and Cordy, D. R. (1981): Olfactory neuroblastoma in a heifer. Vet. pathol. 18, 536-540

Berger, L., Luc, G., et Richard, D. (1924): L'esthésioneuroépithéliome olfactif. Bull. Assoc. Franc. Etude Cancer 13, 410-421

Cohrs, P. (1952): Infektiöse Adenopapillome der Riechschleimhaut beim Schaf. Z. Krebsforsch. 58, 682-692

Cohrs, P. (1970): Lehrbuch der speziellen pathologischen Anatomie der Haustiere. 5. Auflage, Teil 1, S. 229-230, VEB Gustav Fischer Verlag, Jena

Gerard-Marchant, R., and Micheau, Ch. (1965): Microscopical diagnosis of olfactory esthesioneuromas: General review and report of five cases. J. Nat. Cancer Inst. 35, 75-82

Herrold, K. M. (1964): Induction of olfactory neuroepithelial tumors in Sy. rian hamsters by diethylnitrosamine. Cancer 17, 114-121

Magnusson, H. (1916): Endemische Geschwülste im Siebbein. Z. Inf.Krankh. Haust. 17, 329, ref. in Ellenberger, W., und Schütz, W.: Jahresbericht über die Leistungen auf dem Gebiete der Veterinär-Medizin, Bd. 36, S. 41-42, Verlag von August Hirschwald, Berlin (1919)

Noltenius, H. (1981): Systematik der Onkologie. Klassifizierung - Morphologie - Klinik, Bd. 1, S. 500-502, Verlag Urban und Schwarzenberg, München - Wien - Baltimore

Obert, G. J., Devine, K. D., and McDonald. J. R. (1960): Olfactory neuroblastomas. Cancer 13, 205-215

\section{Dr. Gerhard Loupal,}

Institut für Pathologie und Gerichtl. Veterinärmedizin,

Veterinärmedizinische Universität,

Linke Bahngasse 11,

A-1030 Wien
1952 und 1970). Obwohl alle diese Tumoren ihren Ursprung in der Riechschleimhaut haben, scheinen sie, soweit dies aus den Beschreibungen ersichtlich ist, keine neuroepithelialen Geschwülste zu sein.

Osamura, R. Y., and Fine, G. (1975): Ultrastructure of the esthesioneuroblastoma. Lab. Invest. 32, 454

Osamura, R. Y., and Fine, G. (1976): Ultrastructure of the esthesioneuroblastoma. Cancer 38, 173-179

Pospischil, A., und Dabme, E. (1981): Neuroepitheliale (aesthesioneurogene) Tumoren der Riechschleimhaut bei der Katze. Eine licht- und elektronenmikroskopische Untersuchung. Zbl. Vet. Med. A 28, 214-225

Skolnik, E. M., Massari, F. S., and Tenta, L. T. (1966): Olfactory neuroepithelioma. Arch. Otolaryng. 84, 644-653

Stünzi, H., and Hauser, B. (1976): Tumors of the nasal cavity. Bull. World Health Organ. 53, 257-263

Taxy, J. B., and Hidvegi, D. F. (1977): Olfactory neuroblastoma. An ultrastructural study. Cancer $39,131-138$

Taxy, J. B., and Battifora, H. (1980): The electron microscope in the study and diagnosis of soft tissue tumors. In: Trump, B. F., and Jones, R. T.: Diagnostic electron microscopy, Vol. 3, S. 97-174, John Wiley and Sons, Inc., New York - Chichester - Brisbane - Toronto

Thomas, C. (1965): Durch Nitrosamine experimentell erzeugte Nasenhöhlentumoren bei der Ratte. Verh. dtsch. Ges. Path. 49, 245-248

Wilander, E., Nordlinder, H., Grimelius, L., Larsson, L.-I., and Angelborg, C. (1977): Esthesioneuroblastoma - histological, histochemical and electron microscopic studies of a case. Virchows Arch. A Path. Anat. Histol. $375,123-128$ 